\title{
Bio-mimetic design for architecture built by 3D robotic printing
}

\author{
Alexis Salinas Arriagada \\ Universidad San Sebastián | DGNL STUDIO | Chile | a.salinas@dgnlstudio.cl \\ Rodrigo García Alvarado \\ Universidad del Bío-Bío | Chile | rgarcia@ubiobio.cl \\ Patricio Carrasco Perez \\ Universidad del Bío-Bío | DGNL STUDIO | Chile | p.carrasco@dgnlstudio.cl
}

\begin{abstract}
This work presents a parametric development for Architecture by 3D printing, through robotic arms. It addresses a bio-mimetic design approach based on morphological features of animal beings, and develops a spectrum of design possibilities for vertical pieces. The measurements arising from the process extrusion and the possibilities of eccentricity of the manufacturing cord are exposed. As well as variables for design, like the own gravitational restrictions and vertical growth, both in morphological and constructive logic, as a search for relationships closer to the natural world. Suggesting that the new deposition construction systems call for an architecture based on biological principles.
\end{abstract}

Keywords: Architecture; Parametric Design; Bio-mimetic; Robotics; 3D-Printed Construction.

\section{INTRODUCCIÓN}

Este trabajo presenta un desarrollo de Diseño Paramétrico enfocado en la Arquitectura por impresión 3D, ejecutada mediante brazos robóticos. Aborda una óptica de diseño basado en la mimesis de seres animales, específicamente Moluscos Bivalvos, Escafópodos y Crustáceos Cirrípedos de la familia Balanidae. Describe el proceso desarrollo orientado a ampliar el espectro de posibilidades formales en elementos verticales cilíndricos, propendiendo progresivamente a comprender y establecer la relación entrea la superficie y el cordón de impresión. Considerando el comportamiento del cordón de impresión; como materia y elemento constructivo, como generador y posibilitador del diseño. Se expone la data y las mediciones surgidas del proceso, la capacidad de extrusión y las posibilidades de excentricidad del cordón de impresión, como variables para desafiar restricciones gravitacionales propias de erguirse en vertical desde un diseño generado desde lo morfológico como desde su lógica constructiva, en una búsqueda relaciones más próximas al mundo natural.

De manera similar los proyectos Pylos de IAAC y Concrete Choreagry de ETH, han desarrollado elementos verticales impresos; orientados en el primer caso a probar materiales (http://pylos.iaac.net/), y en el segundo caso a explorar formas innovadoras (Anton et al, 2020). En ambos casos, utilizando simetrías radiales en un eje central, para generar diversos perfiles dentro de un área horizontal. Con el fin de determinar posibilidades materiales y expresivas, sin manifestar su vinculación paramétrico con las capacidades resistentes. En este trabajo se plantea una metodología de diseño para envolventes impresas, probadas en elementos verticales cilíndricos y tubulares, para verificar su factibilidad producción y extensión, basado en una analogía biológica.
Este trabajo formula una óptica original para las últimas tecnologías de manufactura robotizada por adición material, formula generar un nuevo enfoque de diseño y construcción, para lógicas impresión 3D basada en los códigos formales de crecimiento, la textura y la expresión natural de los organismos biológicos, proponiendo generar una Diseño Arquitectural más próximo al lenguaje de la Naturaleza.

\section{LA ARQUITECTURA Y RELACIÓN CON LA NATURALEZA.}

La relación con la naturaleza es una referencia recurrente en arquitectura, desde la mímesis o imitación de las proporciones y figuras que promovían la cultura clásica, hasta los llamados al origen de Gaudí y sus construcciones Fito-Mórficas. La racionalización constructiva parece oponerse al organicismo, especialmente durante el movimiento moderno, que impulsó la regularidad y abstracción, relegando las formas complejas y las analogías naturales a situaciones causales. Los conceptos naturales utilizados en la arquitectura suelen ser formas generales que aluden más a simbolismos que a procesos biológicos. Se suelen ocupar figuras similares a elementos naturales, pero realizadas con técnicas y materiales constructivos convencionales, de modo que la referencia se queda en el vocabulario exterior, la alegoría.

Los procesos constructivos actuales se basan en elementos semi-industrializados con materiales procesados para adecuarlos a una producción masiva y repetitiva, basado en el ensamblaje en obra de los productos, con mucha faena de modo artesanal. El enfoque natural es radicalmente distinto, ya que inicia con 
la utilización de recursos del lugar y procesos de crecimiento basados en operaciones fisicoquímicas $\sin$ esfuerzos externos, de modo que la energía empleada es mínima y adaptada a la situación local, y a las necesidades de los organismos. Esta analogía profunda con la naturaleza no ha sido considerada en la arquitectura, al basarse en procesos regularizados de construcción.

Este trabajo propone una óptica distinta para la impresión robótica para arquitectura; impulsando un nuevo enfoque de diseño y construcción, basado en el crecimiento natural de los organismos biológicos. De modo de generar una Arquitectura más conectada a lo inherentemente natural del mundo que habitamos, que tenga el potencial de surgir de la situación local y acoger los requerimientos con coherencia, formal y material.

\section{LA IMPRESIÓN 3D, ANALOGÍAS CON MOLUSCOS Y CRUSTÁCEOS.}

La lógica de la impresión 3D, se comprende por la deposición sucesiva en capas de un material en estado inicial líquido, cuya condición obedece a la de un fluido no newtoniano. Una propiedad fundamental posterior a su estado inicial de maleabilidad es la de solidificarse al paso de un lapso tiempo, aspecto que depende de la composición del material a solidificarse en cuestión. El resultando de la sucesiva deposición de capas individuales del material genera un objeto monocasco de condición constructiva total y unitaria, estructuralmente en equilibrio, un elemento capaz de resistir cargas adicionales a sí mismo.

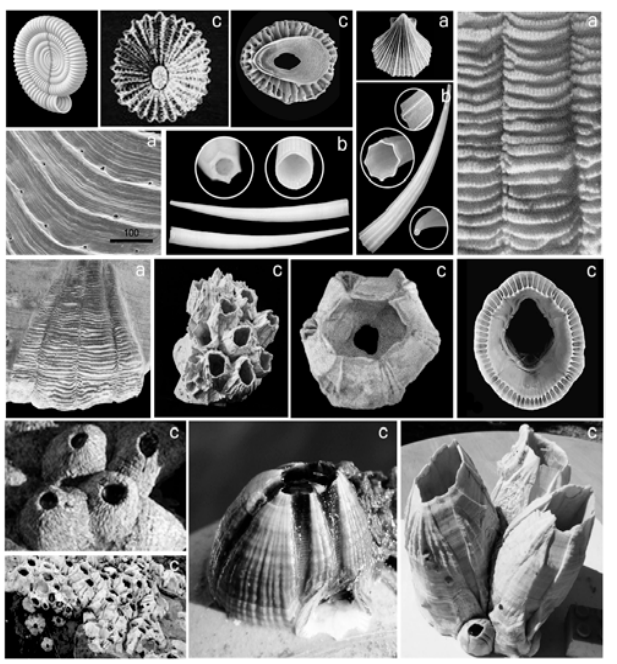

Figura 1:

(a) Bivalvos, (b) Escapófodos, (c) Crustáceos Cirrípedos

En la naturaleza existen seres conformados por caparazones y conchas. Los pertenecientes al mundo marino; molusco y crustáceos presentan una condición similar, su resistencia supera ampliamente el peso propio, presentándose como sujetos dignos de referencia y estudio en su morfología, lógica de desarrollo, y estructura permitiendo establecer analogías con el proceso de impresión 3D, invitando entonces a la observación y análisis de ellos para la adquisición y comprensión de parámetros que permitan e inspiren el diseño, buscando también en ello dilucidar un camino lógico y un lenguaje propio para esta emergente metodología constructiva, cuyo potencial se posiciona en las fronteras del Diseño Arquitectónico.

\section{METODOLOGÍA.}

Este trabajo se basa en la observación morfológica, superficial, textural y en la sección de forma, infiriendo reglas y lógicas geométricas implicadas en la conformación de corazas y caparazones de ; Moluscos Bivalvos, Escapófodos y Crustáceos Cirrípedos, enfatizando en estos últimos avanzado el desarrollo.

Por medio de la comprensión de la composición geométrica, interpretar y volcar las conclusiones, como insumos argumentales para el desarrollo de algoritmos generadores de modelos formales, dilucidando $y$ estableciendo reglas relacionales para el Diseño Parametrizado de elementos arquitectónicos verticales, preponderantemente tubulares cilíndrico, pertinentes y compatibles a la deposición de mezclas cementícias para la Impresión 3D. para ejecutarse con un equipamiento Robótico; Brazo KUKA120 R2500PRO, en secciones horizontales seriadas verticalmente como perfiles de forma, desarrolladas y programadas en los softwares Grasshopper/Rhinoceros. Adquirir en ello el dominio de las variables fundamentales, que permitan generar, fabricar y desarrollar alternativas morfológicas que profundicen progresivamente en la incorporación de lo observado de las especies biológicas, como sujetos de estudio, propendiendo a una mayor asimilación de ellas como ADN de Diseño y proposición de principios morfológicos, que enriquezcan el imaginario y el abanico de posibilidades en la impresión tridimensional y con ello abrir nuevas oportunidades para el campo del Diseño de Arquitectónico.

\section{DESARROLLO.}

\section{OBSERVACIÓN Y ANÁLISIS.}

Las características observables, más allá de aquello que es lógico, como el material solidificable depositado en capas sucesivas, se encuentra la condición de esas capas sucesivas, como una superficie total, que presenta un grado de espontaneidad textural y rugosidad, que entendemos como aparentes imprecisiones modulares, juiciadas generalmente como azarosidad o aleatoriedad, sin embargo ha de entenderse con otra profundidad, si nos detenemos y pensamos en ellas como en otros niveles de organización y orden comprendiéndolas como; espectro de libertad modular, infiriendo en ello parámetros como: Rango, Dominios, Límites y Patrones, indicaciones que resultan sustantivas para la asimilación de esas características como aspectos inherentes a los seres de estudio, . Una característica adicional, es la capacidad de erguirse contra la gravedad, limitación que la impresión 3D también ha de sortear. Solo la especie de Crustáceos Cirrípedos, Fig. 1 (c) posee tal característica de modo significativamente mayor, además de una continuidad parcial de su patrón textural superficial, constatando 2 zonas, una de rugosidad vertical y otro patrón horizontal sin esa rugosidad, como un valor lógico/formal diferenciador, eso como evidencia de su lógica de crecimiento, implicando en ello una comprensión de resistencias diferenciadas por zonas o tramos. 


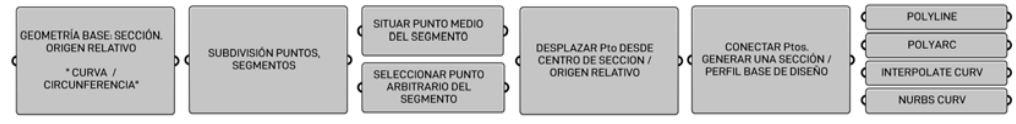

\section{1ra. GENERACIÓN DE DISEÑO}

Generación desde Observación de Morfología General
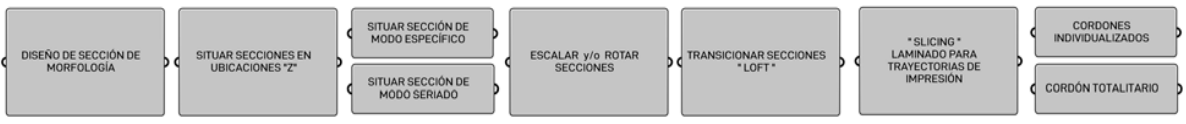

Figura 2: Esquema conceptual de algoritmo para la 1ra. Generación de Diseño.

\section{GENERACION DE UN PLANTEAMIENTO PARA EL DISEÑO DE MORFOLOGÍAS VERTICALES.}

Inicialmente se obtienen principio generales, que permitan argumentar el origen referencial del Diseño, infiriendo de los sujetos de estudio, las secciones transversales de ellos como perfiles morfológicos elementales para la generación del mismo, el que se origina y plantea desde una geometría basal, circunferencia, posicionada en un punto en el espacio tridimensional ( origen relativo), que mediante subdivisiones por puntos en ella, generará segmentos, de los cuales mediante la obtención de un punto medio se desplazará desde el centro, conectándose estos nuevos puntos, con aquellos generados previamente en la subdivisión, mediante un componente de curvas "polyline", "polyarc", "interpolate curv", "nurbs curv" para confeccionar en ello un perfil base de diseño. (Fig 2; Perfil Base Diseño)

Desde el perfil base de diseño, el procedimiento siguiente es la conjugación de esa geometría matricial, con acciones de desplazamientos, " $Z$ ", escalamientos y/o rotaciones, ambas de modos específico o seriado progresionalmente, obteniéndose un primer algoritmo de diseño referenciado de modo temprano con lógicas morfológicas de seres marinos basados en conchas y caparazones. (Fig 2; $1^{\text {era }}$ Generación de Diseño.)

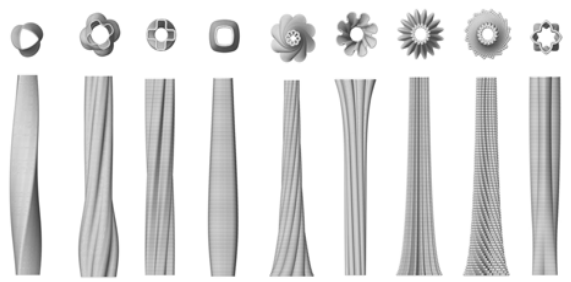

Figura 3: Columnas de referencias seccionales $1^{\text {ra }} \mathrm{Gen}$.

Plantear los parámetros elementales para la sucesión de capas/cordones para Impresión "Laminado" ( slicing ), cuya altura entre si ( hs: Slicing ), es homóloga al diámetro del terminal de impresión (Boquilla), a la que debe sustraerse una dimensión de contacto entre cordones ( $h$ : contacto (hc)), que según consistencia de mezcla poseerá un rango de entre 2 a $5 \mathrm{~mm}$, condicionado eso directamente por el material de impresión, que en esta investigación obedece a una mezcla cementícia " Micro Hormigón " cuyo terminal en uso es de $25 \mathrm{~mm}$, por tanto el h: Slicing sustrayendo h contacto ( hc: $3 \mathrm{~mm}$ ), resulta en un valor de; hs: $22 \mathrm{~mm}$.

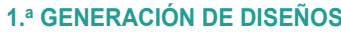

El procedimiento posterior a la confección de perfil base de diseño, es la conjugación de esa geometría matricial, con acciones de desplazamientos y seriaciones en " $Z$ ", escalamientos $\mathrm{y} / \mathrm{o}$ rotaciones, específicas o seriadas progresionalmente, obteniéndose un primer algoritmo, y los primeros diseños referenciados de modo sutil y temprano con las lógicas morfológicas composicionales y texturales de seres marinos basados en conchas y caparazones. Plantear el diseño desde este punto, no garantiza la factibilidad y el éxito de impresión del mismo, ha de comprenderse entonces el comportamiento de la materia cementícia propia de impresión mediante pruebas iniciales.

\section{PRUEBAS INICIALES DE LA MATERIA.}

Comprender el comportamiento de un material con propiedades conocidas como el mortero, aproximación estrecha frente a la mezcla "Micro Hormigón"; un mortero enriquecido con componentes adicionales al cemento, que para impresión se manipula particularmente en una condición de absoluta libertad como fluido , sin moldajes ni contenedores pre formativos, que es como el paradigma constructivo domina el hormigón. Eso provee preguntas nuevas y esenciales para el dominio y control de la tecnología de impresión 3D y su técnica constructiva hormigón.

La primera generación de diseño presenta morfologías tempranas de baja complejidad, que ya proponen una dificultad al momento de evaluar el comportamiento del material de impresión, la torsión y la reducción progresiva
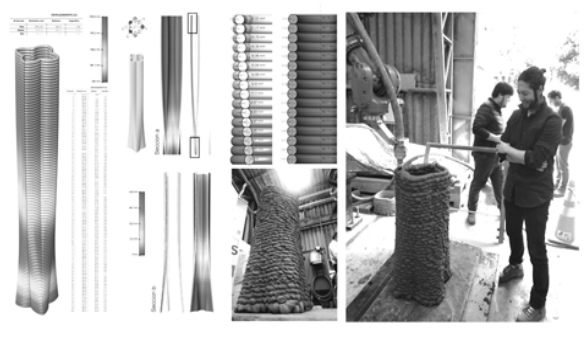

Figura 4: incorporación de la data generada y su impacto en la generación de diseño y su resultado en impresión. 
de la sección de diseño, dificulta la comprensión de las razones de éxito o fracaso de las primeras pruebas, dificultando el concluir si sus razones obedecen a su morfología o al comportamiento del material. La impresión inicialmente debió optar por algunas morfologías abstractas lo que permitió realizar y responder esas preguntas fundamentales y simples para la comprensión de fenómenos medulares de base, para posteriormente complejizar la combinatoria entre materia y forma.

\section{RESPUESTAS FUNDAMENTALES DE LA FABRICACIÓN PARA LA CONSIDERACIÓN EN EL DISEÑO}

Condicionadas desde un material de impresión "Micro Hormigón" de características locales, las primeras respuestas son:

Diam Terminal Impresión ..... D= $\mathbf{2 5} \mathrm{mm}$

h Contacto Slicing .............hc $=5 \mathrm{~mm}$

h Slicing ....................... hs $=20 \mathrm{~mm}$

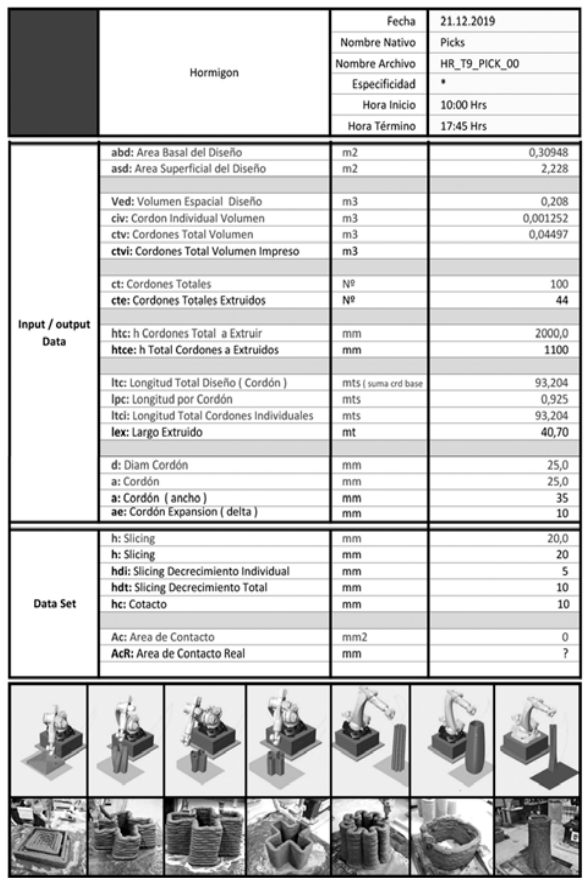

Figura 5: Extracción de Datos Generados de última prueba ( Fig. 4) de impresión Micro-Hormigón escala 1:1.

Primariamente existe un asentamiento en los cordones de impresión, posteriormente ello es compensado con el $\boldsymbol{h}$ de contacto, (hc) entre cordones, la incorporación de tal variable, presenta un asentamiento no evidenciable ocularmente, al comprobarse por medición una distancia estable entre el cordón en extrusión y terminal de salida.

¿Cuánta es la libertad de torsión en un diseño?: El micro hormigón desarrollado, de momento no permitió torsionar el diseño, ha de desarrollarse una capacidad de fraguado en menor tiempo para ello, o abordar la impresión por tramos, implicando una pérdida de trabajabilidad del material en los depósitos de bombeo. Sin embargo es una condición temporal no limitante, bastará establecer otras condiciones de mezcla para sortear esa dificultad.
¿Es posible expandir el perímetro de cordón de impresión?, Desplazando el cordón desde su eje de gravedad, $1.65 \mathrm{~mm}$ respecto a su cordón antecesor, permite lograr un mayor grado libertad formal al momento de plantear e indagar otras complejidades de diseño.

La capacidad de levantar cordones sucesivos es condicionada por 2 factores; el comportamiento de la materia de impresión, su tiempo de fraguado y solidificación -el aglomerado- y la velocidad con la que los cordones se depositan sucesivamente, propiciando en ello alta carga entre sí en un reducido margen de tiempo. Para sortear de modo favorable tal realidad, se establece una velocidad "crucero" de trayectoria de impresión de 0.15 $\mathrm{mt} / \mathrm{seg}$.

El proceso de fabricación por impresión 3D Robotizada, demanda la generación de un código de interpretación de las trayectorias para la deposición de la materia imprimible, en un lenguaje propio del equipamiento para impresión, Robot KUKA120 R2500PRO. Este código es la identificación de posiciones relativas, puntos relativos, en el espacio tridimensional propios del seccionado de la morfología a imprimir -laminado "slicing"- generado del diseño en el entorno virtual computacional, cuya manifestación tridimensional en el mundo real analógico, se realiza mediante la deconstrucción del laminado en esos puntos en el espacio, proceso que se realiza en el software de gestión de movimiento del brazo robótico , plugins para Grasshopper; KUKA PRC, cuyo formato de salida es un archivo de extensión *.src que provee de esas posiciones como un total contínuo y consecutivo leídos como líneas, "Command Line" , "Command Spline" (setear en Kuka PRC). Se comprende en esta operación una relación importante, la fidelidad interpretativa de esos puntos para la regeneración en el espacio analógico del slicing y con ello la morfología diseñada para dar resultado finalmente a su homóloga fabricada. Se establece que; a mayor densidad de la deconstrucción del slicing, mayor fidelidad del diseño digital, pero será menor capacidad de aceleración del brazo robótico en su ejecución, puede entenderse aquí que los procesos futuros de optimización de tiempo pueden verse afectados y/o anulados bajo esta condición.

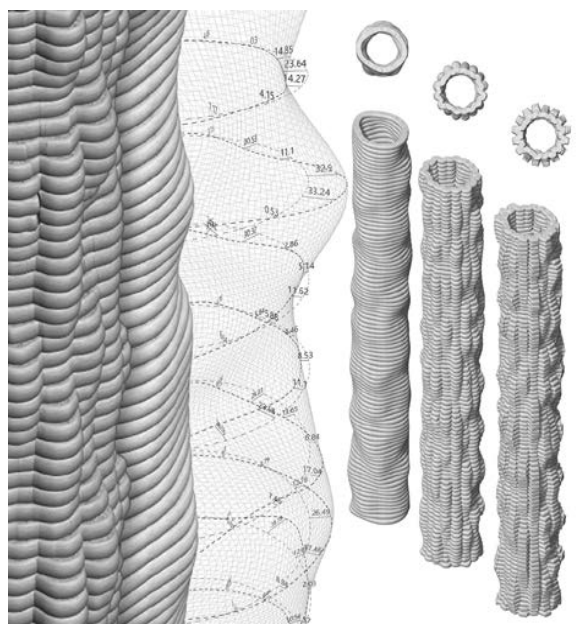

Figura 6: 2da Generación de Diseño. Incorporación de lógicas Texturales de Variabilidad Perpetua 
2da. GENERACIÓN DE DISEÑO

Observación desde Lógica Morfológica Expresiva

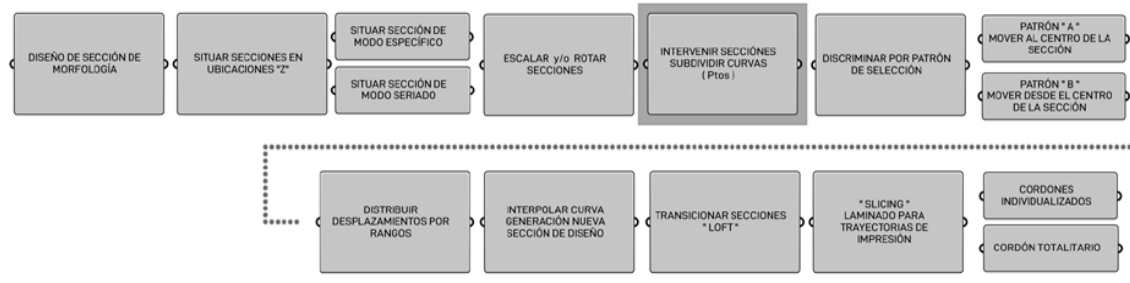

3ra. GENERACIÓN DE DISEÑO

Observación desde Lógica Morfológica Expresiva / Ejemplar Específico Austromegabalanus Psittacus / Familia Balanidae
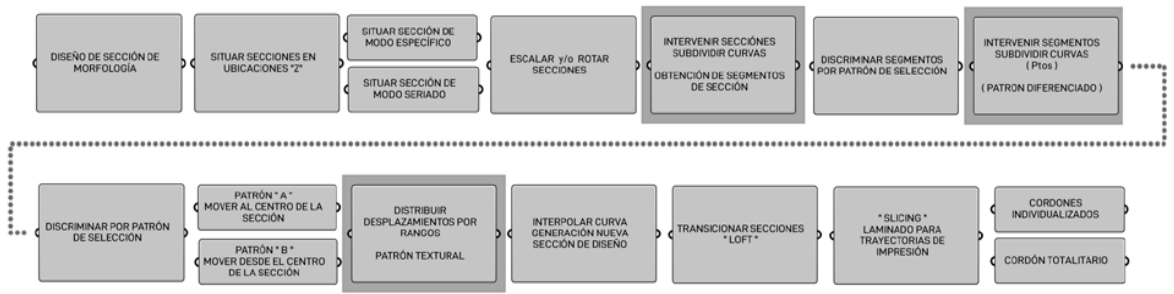

Figura 7: Esquema conceptual de algoritmo para 2da. y 3ra. Generación de Diseño.

Los modelos iniciales de baja complejidad formal y textural se han establecido con una fidelidad de trayectoria de 20 $\mathrm{mm}$, posteriormente tal sentencia se ha planteado en 10 $\mathrm{mm}$ entre puntos, ello ha sido directamente condicionado por la complejidad del diseño y el detalle del mismo, condicionando la velocidad de ejecución, sin embargo este aspecto puede sortearse con un terminal de impresión reducido, generando un cordón de mayor precisión, ello demandará una mezcla cementicia apropiada o ajustar otros aspectos como el empuje de la mezcla. El problema puede ser inexistente si por la envergadura del diseño se provee una deconstrucción más espaciada entre puntos del slicing no afectando en ello comprensión del diseño.

\section{2. ${ }^{a}$ GENERACIÓN DE DISEÑOS.}

Una segunda observación y análisis a los ejemplares de estudio, permite concientizar la semejanza entre sí como especie, presentando variabilidad entre individuos sin dejar de comprenderse como perteneciente a la misma, pero que sin embargo logran entenderse como individuos identificables; por dimensión, proporción, o robusticidad. Existe otra condición propia de moluscos y crustáceos cirrípedos, un segundo nivel de complejidad presente en la expresión superficial de sus conchas y caparazónes, entendiéndose como patrones de diseño inherentes a su lógica conformacional por capas sucesivas de " conquiolina", que posiblemente por diferentes densidades en el depósito del material, por el tiempo de flujo del mismo o por variaciones de corrientes de agua en el contexto marino, presentan perpetua variabilidad en su impronta textural.

La data obtenida en las última pruebas de material, Fig.5 sumada a una segunda observación y un análisis de mayor profundización, permitió desarrollar una nueva generación de diseños que considera esa información para la incorporación de nuevas variables aplicables a los mismos, lo que permitió generar nuevas cualidades formales y una resultante expresiva mucho más próxima a lógicas miméticas como analogías a seres conformados por caparazones y conchas.

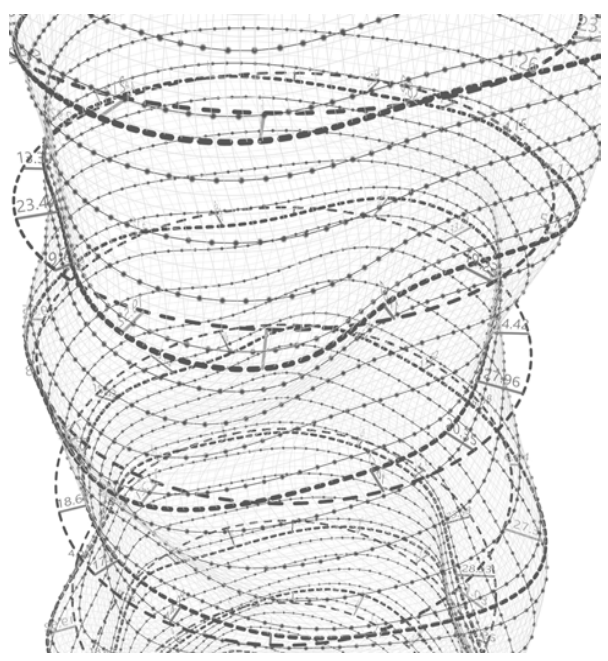

Figura 8: Secciones de 2da Generación, establecidas por rangos de movilidad al interior y exterior de una sección de primera generación, trazada posteriormente con curvas Nurbs, cuya transición en superficie, expresada en malla (mesh). Se grafican los puntos de trayectoria y la misma.

El método de 2da generación de diseño incorpora un aspecto fundamental, una variabilidad perpetua, consiste en establecer secciones especificas o seriadas que 
definan la morfología cilíndrica tubular primaria, estableciendo posiciones locales desde la cuales se generarán desplazamiento mediante rangos de movilidad o libertad para los atributos de variación, Fig 8 que deben ser distribuidos de modo particular para cada condición de localidad ( valor distinto por puntos de modo no progresional o incremental ). El rango poseerá valores positivos y/o negativos, 0 vectores de direcciones opuestos para las posiciones relativas de los puntos, cuya conexión entre si genera nuevos perfiles locales de diseño que en su transición de superficie (loft), proveen de una morfología de segunda generación, incorporando en ella la condición de variabilidad perpetua, Fig 6 (a).

La distancia de la sección del perfil de diseño basal primario seriada en "Z" y la densidad en la subdivisión por puntos de las mismas, división en similar cantidad a todas, garantizará la coherencia y la correlación de empate (maridaje) de los datos. La magnitud de ellos será distintos y particular a cada uno de los puntos y la conexión de ellos mediante estrategias de curvas; "polyline", "polyarc", "interpolate curv", "nurbs curv" proveerán una expresión suavizada o una de rugosidad. La fidelidad del diseño al momento de la impresión estará determinada por el laminado "slicing" de la morfología generada y finalmente por la deconstrucción del trazado para la interpretación del lenguaje robótico en puntos.

Han de distinguirse en el proceso de diseño, las curvas que dan origen a: Las secciones o perfiles de generación de morfología; de la manipulación de ellas emergerá una segunda generación de curvas de perfil de diseño. La resultante entre la Morfología y la intersección de planos relativos $\mathrm{XY}$, situados referencialmente a en el vector " $\mathrm{Z}$ " a la distancia del slicing, generará la curva para trayectoria y finalmente, luego en su deconstrucción, la "curva" de trayectoria para deposición e impresión.

\section{3. a GENERACIÓN DE DISEÑOS}

En los seres sujetos de estudio, se logra comprender que un tipo de ellos, los crustáceos cirrípedos de la familia de los Balánidos, Fig. 1 (c) poseen lógicas formales de interés y pertinencia al este erguirse en mayor verticalidad que los otros géneros de moluscos bivalvos y escapófodos hasta el momento observados Fig. 1 (a), (b). Es desde esa significativa cualidad que se presenta tal criatura para una observación más dedicada, constatando en el lógicas morfológicas relevantes; Una textura superficial como parte de su cualidad característica y lenguaje propio de la naturaleza, aquella que se establece con iteraciones perpetuas que no necesariamente son constantes e idénticas, que en término de diseño tal aspecto se entiende como un patrón con "Jitter", que se traduce como una fluctuación del mismo y que matemáticamente se entiende y traduce en límites, dominios y rangos de libertad en él, ello resulta ser indicativo para el control esa variable expresiva de diseño. Otra característica relevante, es que en una misma materialidad posee una expresión textural diferenciada, ello rompe el paradigma del micro-universo de estudio ( es necesario situarse sólo en el caso de estudio debido al casi infinito universo marino y su rica biodiversidad) de momento y desde lo observado los seres de estudio, y sus amplias familias poseen una lógica expresiva totalitaria e igual, un patrón constante y perpetuo, que en esta familia de crustáceos no se constata de igual modo, se constata por tramos o sectores lo que empuja el pensamiento en analogía y entender en ello 2 tipos de pared o muro extruido, ello resulta indicativo a una lógica constructiva con oportunidades en escala 1:1 indicativas para una proto vivienda, presentándose este ejemplar como una invitación ineludible a abordar sus lógicas y contribuir al espectro formal / expresivo para impresión 3D y enriquecer así su lenguaje.

El objeto de diseño generado Objeto Cilíndrico Tubular "Cuerpo Balanus" posee zonas de rugosidad que en la totalidad de la figura se comprenden sugiriendo nervaduras, que podrían comportarse como sectores con mayor capacidad de carga vertical de esfuerzo a la compresión. Aborda y asume la diferenciación textural de la superficie expresiva del balánido, planteando su morfología con un mayor grado de equilibrio y simetría, aspecto que manifiesta el control geométrico como elemento de proposición Arquitectural, contrastando con el aparente descontrol de su textura focalizada y diferenciada, que desde la perspectiva proyectual del diseño, es una variable, en control, subyugada a una dimensión conocida, cuyo rango no supera el límite de estabilidad dilucidado en las pruebas iniciales de hormigón, $40 \mathrm{~mm}$ de rango, distribuidos en 1080 localizaciones. Cada una de ellas asumiendo un valor único dentro de ese rango, en un dominio geométrico de la variabilidad en desplazamiento, cuya dimensión de dominio matemático está entre 10 a $50 \mathrm{~mm}$, se genera y resuelve la expresividad de la superficie "la rugosidad", como una característica cualitativa particular propia de las lógicas de la naturaleza.

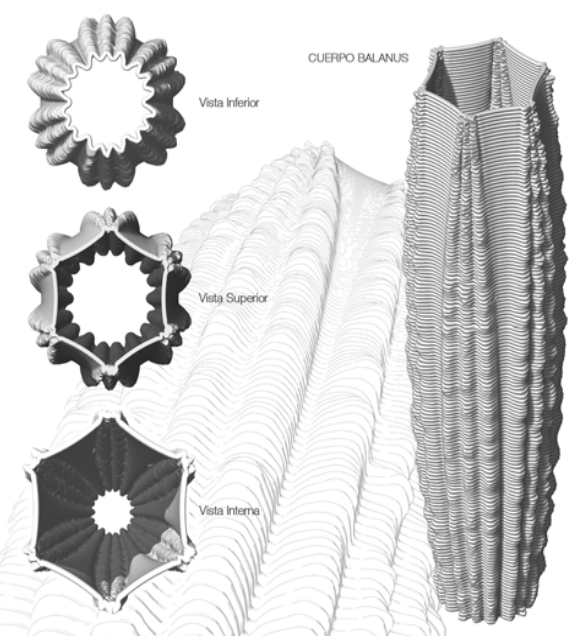

Figura 9: Objeto Cilíndrico Tubular "Cuerpo Balanus". Incorpora lógicas estrechas de ejemplares Crustáceos Cirripedos. ref: Figura 1 (c).

\section{RESULTADOS}

\section{EXPERIENCIA EN IMPRESIÓN EN MICROHORMIGÓN}

En el trabajo con las mezclas cementícias "Microhormigón" se presenta una relación lineal entre la altura de impresión obtenida y la cantidad de material requerido para su impresión, existen variables en el proceso de impresión de una misma mezcla a distintos tiempos óptimos para la utilización del material en una misma velocidad de trayectoria $(0,15[\mathrm{~m} / \mathrm{s}])$, cuya preocupación ha de estar en 
el control del material de extrusión (cantidad de material extruído por unidad de tiempo, [lt/s]).

La gráfica presentación la relación entre la altura de Impresión y la cantidad, la densidad del "Slicing" (laminado del diseño), cantidad de cordones. La gráfica de mayor pendiente posee menor densidad de cordones impresos, en un terminal de $25 \mathrm{~mm}$, la altura de slicing es de $\boldsymbol{h s}: 20$ $\mathbf{m m}$, utilizando aproximadamente 20 Its de mezcla alcanzando una altura de $150 \mathrm{~mm}$. Las gráficas de menor pendiente, muestra que con un terminal de extrusión (Boquilla) de $20 \mathrm{~mm}$ y su respectivo hs: $15 \mathrm{~mm}$ se obtiene una altura de $400 \mathrm{~mm}$, con similar cantidad de mezcla.

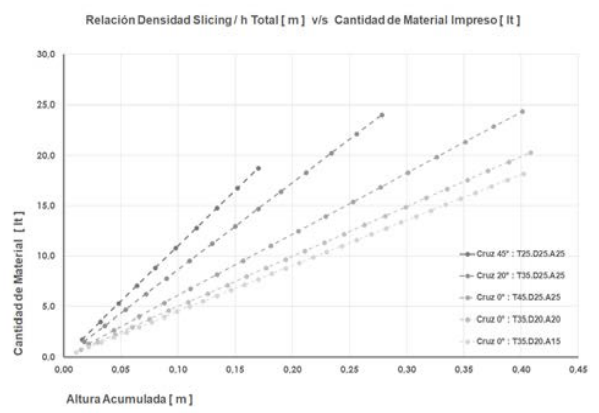

Figura 10: Rendimiento Material

El desarrollo del elemento con una mayor densidad de cordones, es decir una menor altura de slicing, hs:20 mm genera una mayor longitud de trayectoria de cordón de impresión para lograr la misma altura en la figura al poseerse un terminal reducido de 25 a $20 \mathrm{~mm}$, ello implica modificar la velocidad de crucero de trayectoria, otorgando a la mezcla una mayor superficie expuesta por unidad de tiempo, logrando que el material adquiera mayor capacidad de autosoporte para cada cordón como individualidad, como así también mayor autosoporte como elementos colaborantes y totalidad auto soportante.

Una mayor densidad del slicing, menor hs: del laminado, genera una longitud de trayectoria mayor y con ello mayor tiempo de impresión, obteniéndose una relación directamente proporcional referida a la densidad de impresión respecto al tiempo e inversamente proporcional respecto al rendimiento material, a mayor slicing, menor demanda material, siendo esta última combinación de variables una tendencia en el éxito de impresión respecto a la "erguibilidad" de las impresiones, reduciendo la posibilidad de colapso en los elementos impresión antes de culminar el proceso, otorgando además una mayor definición de la forma impresa, presentándose un mayor grado de precisión.

\section{CONCLUSIONES}

La observación, estudio y análisis de seres conformados por caparazones y conchas demuestra ser un universo muy enriquecido de información, referencia e insumo para la comprensión de nuevas lógicas formales y conformacionales de seres cuyos proceso de materialización de cuerpos rígidos, se homologan al proceso constructivo para y por impresión 3D. Adquirir esa información y el dominio de ese conocimiento son aplicables directamente a la generación nuevos diseños, y de mayor pertinencia a la oportunidad y lógica que la nueva tecnología constructiva posee como cualidad y potencial inherente, propias de ser explotadas y potenciadas, cuya limitación hoy es un aspectos técnico, que como todos los problemas técnicos han de resolverse con pruebas ensayos, mediciones y corrobaciones.

La serie pruebas iniciales respecto al hormigón, brindaron información vital que permitió fabricar varios ejemplares prematuros, desembocando en la fabricación de un proto elemento Arquitectónico, ya con un proceso de mayor maduración, permitiendo a este abordar capacidad máxima de los recursos disponibles 90 lts de mezcla sin colapso, en un h 1.10mts de impresión continua, comprobándose la viabilidad de la materialización de lo diseñado, que incorporó la data de las pruebas iniciales, propició la confiabilidad de la factibilidad a favor para el desarrollo de una 2da y 3ra generación de diseños, quienes se subyugan a esas variables como input, estándar para la actual etapa de desarrollo. Cambiar valores de input, terminales de extrusión por ejemplo, afectará la efectividad de ese estándar de la mezcla. La densidad del patrón de diseño o la velocidad de trayectoria, repercutirá directamente en el éxito o fracaso de la fabricación, es por tanto vital generar perfiles de mezcla cuyos comportamientos han de establecerse como un estándar o mezcla tipo, para rangos específicos asimilación por ejemplo:

Mezclas de Cordón más grueso, serán idóneas para diseños en extensión , cordones más finos , para mezclas de elementos en vertical, ambos requieren terminales, boquillas distintas, y con ello extrusión distinta y empuje de mezcla distinto.

La capacidad del material y su comportamiento aún posee camino por analizar y comprobar, además de la comprensión entre cordones de impresión y su patrón de diseño para brindar una mayor oportunidad autosoportante y colaborativa y otorgar cualidades considerables para sortear la fragilidad prematura y la inestabilidad de la deposición inicial en la impresión con materiales cuyo fraguado no es instantáneo y que requieren de un lapso de tiempo para iniciar su solidificación.

\section{DISCUSION}

El universo biológico de nuestro planeta es enriquecido en seres de similares lógica a las presentadas observadas y otras que proponen nuevas ópticas para el desarrollo de estrategias de diseños como así también las de materialización de lo diseñado. Existe una pertinencia innegable en tal arrojo observacional, de ello se garantizan resultados de alta coherencia contextual, y con información nueva, el material implicado en conchas y caparazones, el calcio y conquiolina existen en abundancia. No es de despreciar, y esto es sujeto de comprobación, que una caparazón homologa generada de hormigón no poseería la misma capacidad resistente, que la generada por la naturaleza, posicionado a la solución biológica con alta pertinencia estructural y sin duda una mayor pertinencia medioambiental, entonces sumar actores diametrales como biólogos desde la vereda de observación de morfologías y procesos y químicos e Ing. en construcción situados en el enfoque estructural material abrirá el campo de la Arquitectura a nuevas respuestas para la creación de la realidad del mañana.

El dominio de la capacidad para la generación de diseños cuya motivación nazca de un análisis directo de la 
naturaleza, de sus variables organizacionales, de su proceso conformacional o de su lógica textural, o expresividad, desafía la generación del diseño con un fin más allá de la mera exclusividad de su expresividad, plantea el desafío de la correcta inferencia y compresión en las matemáticas implicadas en ello, desafiando así mismo la propia capacidad del estado del diseño y del diseñador como generador de realidad abriendo las posibilidades al desafiar los límites de aquello que puede diseñarse y construirse por esta emergente tecnología disponible.

La capacidad de la fabricación asistida de forma computacional no establece diferencias para la materialización de objetos y formas disimiles, la fabricación computacional robotizada y el desarrollo de esta mediante el sistema paramétrico, conserva intacto ese principio, es aquí donde aparece otra dimensión de observación aplicable a las lógicas de impresión 3D. Mas allá de cualidades materiales o formales existen en la naturaleza dinámicas y lógicas organizacionales puntualmente en el caso de estudio. Los crustáceo cirrípedos, se agrupan por colonias, variadas en tamaño, posición, distribución , agrupación y forma, si esos principios estuviese abordados por la impresión 3D para la generación de conjuntos volumétricos como las viviendas, ello no demandaría un costo adicional por la particularidad de un diseño, o algún aspecto a variar en ello, presentándose esta proposición como una manera de dar respuesta diferenciada a la diversidad de formas del habitar presentando una oportunidad de respuesta que hasta el momento el sistema convencional de fabricación por partes y piezas no puede responder a ese requerimiento, será solo desde la impresión tridimensional y versatilidad frente a la variabilidad que se proveerá de nuevos lenguajes y códigos para la Arquitectura.

\section{REFERENCIAS}

Meredith, M. (2008). From control to design : parametric, algorithmic architecture. Barcelona: Actar.
Woodbury, R. (2010). Elements of parametric design. Londres: Routledge.

Gürsel Dino, I. (2012). Creative design exploration by parametric generative systems in architecture. METU: JFA, 29(1), 207-224. Jabi, W. (2013) Parametric design for architecture London: Laurence King

Wortmann T, Tunçer B, (2017) Differentiating parametric design: Digital workflows in contemporary architecture and construction, Design Studies 52, 173-197

Dai, Rushi, Ethan Kerber, and Sigrid Brell-Cokcan. 2019. "Robot Assisted Assembly of Steel Structures." Intelligent and Informed Proceedings of the 24th International Conference on ComputerAided Architectural Design Research in Asia, CAADRIA 2019 1: 163-72.

Allgaier, Christoph et al. 2019. "Snails as Living 3D Printers: Free Forms for the Architecture of Tomorrow." Biomimetics for Architecture (June): 126-33.

Bonwetsch, Tobias. 2012. "Robotic Brickwork ROBmade Commercial."

Fischer T and Herr C (2016) Parametric Customisation of a 3D Concrete printed Pavilion, CAADRIA 2016 549-558:

Y. Ahmed, Zeeshan, Freek P. Bos, Rob J.M. Wolfs, and Theo Salet. 2016. "Design Considerations Due to Scale Effects in 3d Concrete Printing." 8th International Conference of the Arab Society for Computer Aided Architectural Design: 1-10. http://repository.tue.nl/856943.

Andaman Sea and the Gulf of Thailand." Zoosystematics and Evolution 93(1): 13-34.

Raspall F and Bañon C (2016) vMESH : How to print Architecture? SIGRADI 394-400

Pochai, Ashitapol, Sutin Kingtong, Woranop Sukparangsi, and Salinee Khachonpisitsak. 2017. "The Diversity of Acorn

Barnacles (Cirripedia, Balanomorpha) across Thailand's Coasts: The Andaman Sea and the Gulf of Thailand." Zoosystematics and Evolution 93(1): 13-34. 